\title{
Real-time Notification and Response During Pandemics
}

\author{
Achraf Othman \\ Mada Center
}

Emergency notification during pandemics is part of broader risk management, company-wide communication, and emergency planning strategies, to improve communication, workflow, and services before, during, and after an emergency. As it is very important that the message is broadcasted to everyone including persons with disabilities and Elderly'.

Nowadays and due the current pandemic of COVID-19 as an example, governments and organizations are putting in place systems and applications to alert people of any danger that can impact them. Generally, this is done via a public service messaging system.

In this article, we will go through different innovative approaches to implement a service messaging system that is accessible for everyone. The service automates necessary actions like sending out mass notifications, sharing information, and mobilizing teams to prevent operational disruptions and quicken emergency response. Emergency notification systems are personalized to the requirements and needs of whatever organization implements the software. Emergency notification software can integrate with or feature GIS to create maps and documents as visual aids during crises.

The system is based on Mathematical and simulation models which may be used to inform policy in the early stages of an infectious disease outbreak by evaluating which control strategies will minimize the impact of the epidemic (Probert et al., 2018). In these early stages, significant uncertainty can limit the ability of models to provide accurate predictions, and policymakers do not have the luxury of waiting for data to alleviate this state of uncertainty.

The mass notification system sends audio, text and images to mobile and on-premises devices (Othman et al., 2008). It increases the speed, reach, and success rate of emergency notification communications to get critical information to the people who need it. The information is broadcasted through different channels and in an accessible format to all persons. For example, blind people can access the notification via audio or braille displays, and persons with hearing impairments can use images or short video clips to access the same information.

\footnotetext{
${ }^{1}$ https://www.g2.com/categories/emergency-notification
} 
Real-time notification and response system during pandemics is one of the use cases presented in the Mada Innovation Program under the Culture domain. The program encourages innovators and entrepreneurs to develop innovations that can improve the response for persons with disabilities and elderly during pandemics.

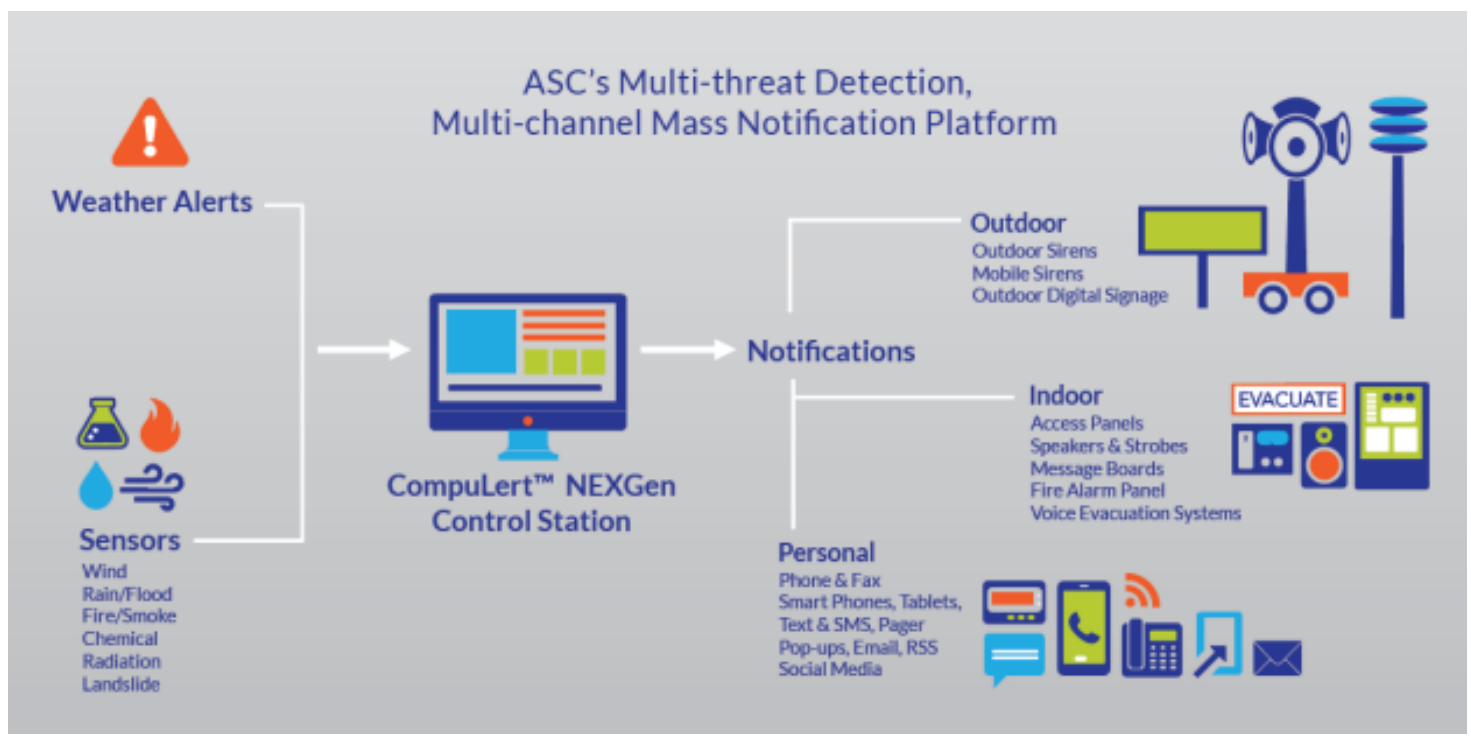

Fig 1. Example of real-time notification system architecture

This can be supported by the program through several streams. Mada works to enable equal basis for PWDs and the elderly to take part in social and cultural life in line with the Arabic identity via ICT. The Center also supports the provision of opportunities for PWDs and the elderly to use their creative, artistic an intellectual potential independently ${ }^{2}$ (Al Thani et al., 2019).

\section{References}

Al Thani, D., Al Tamimi, A., Othman, A., Habib, A., Lahiri, A., \& Ahmed, S. (2019, December). Mada Innovation Program: A Go-to-Market ecosystem for Arabic Accessibility Solutions. In 2019 7th International conference on ICT \& Accessibility (ICTA) (pp. 1-3). IEEE.

Othman, A., El Ghoul, O., \& Jemni, M. (2010, July). SportSign: a service to make sports news accessible to deaf persons in sign languages. In International Conference on Computers for Handicapped Persons (pp. 169-176). Springer, Berlin, Heidelberg.

Probert, W. J. M., Jewell, C. P., Werkman, M., Fonnesbeck, C. J., Goto, Y., Runge, M. C., Sekiguchi, S., Shea, K., Keeling, M. J., Ferrari, M. J., \& Tildesley, M. J. (2018). Real-time decision-making during emergency disease outbreaks. PLoS computational biology, 14(7), [e1006202]. https://doi.org/10.1371/journal.pcbi.1006202

\footnotetext{
${ }^{2}$ www.mip.qa
} 